\title{
Fatores de risco associados ao câncer de mama em uma amostra de mulheres participantes de uma universidade da terceira idade
}

\author{
Fernanda Bussolotto*, Josiane Siviero**, Ana Carolina Pio da Silva***
}

\section{Resumo}

O câncer de mama é o tipo de câncer mais comum entre as mulheres, sendo considerado uma doença de extrema importância para a saúde pública em nível mundial. Acredita-se que sua incidência e mortalidade ascendentes estejam relacionadas à maior exposição a fatores de risco e ao envelhecimento populacional. O objetivo deste estudo foi descrever a presença de fatores de risco associados ao câncer de mama em mulheres participantes da Universidade da Terceira Idade da Universidade de Caxias do Sul - RS. A pesquisa foi do tipo transversal, descritiva e analítica, sendo a amostra constituída por 45 mulheres, com idade entre 51 e 86 anos. A coleta de dados aconteceu no período de março a setembro de 2010. Foram obtidos dados socioeconômicos, reprodutivos, antropométricos, de estilo de vida e do consumo alimentar. As participantes apresentaram um perfil de elevada renda e escolaridade. A idade média da menarca foi de 12,9 $\pm 1,9$ anos e da menopausa, 49,1 \pm 5,4 anos; $62,2 \%$ amamentaram por menos de um ano; 33,4\% foram classificadas como obesas, segundo o IMC; $17,6 \%$ praticavam atividade física regularmente. As mulheres obesas apresentaram significativamente mais fatores de risco que as outras. De maneira geral, a alimentação mostrou-se adequada, principalmente quanto ao consumo diário de frutas, verduras e produtos integrais e à baixa ingestão de gorduras. Apesar disso, o estudo chama a atenção para a elevada prevalência de sobrepeso e obesidade na amostra, fatores que podem ser alterados em nível individual por meio de intervenções de educação e orientação nutricional.

Palavras-chave: Envelhecimento. Câncer de mama. Fatores de risco. Estilo de vida. Dieta.

* Acadêmica do curso de graduação em Nutrição da Universidade de Caxias do Sul. Endereço para correspondência: Rua Antonio Tomedi, 157, Bairro Santo Antão, CEP: 95700-000, Bento Gonçalves - RS. E-mail: fernanda.bussolotto@terra.com.br.

** Nutricionista. Doutora em Ciências da Saúde pela Pontifícia Universidade Católica do Rio Grande do Sul. Mestra em Gerontologia Biomédica pelo Instituto de Geriatria e Gerontologia - Pontifícia Universidade Católica do Rio Grande do Sul. Docente do curso de Nutrição da Universidade de Caxias do Sul e da Faculdade Cenecista de Bento Gonçalves.

*** Nutricionista. Mestra em Ciências Médicas: Nefrologia pela Universidade Federal do Rio Grande do Sul. Docente da Universidade de Caxias do Sul, da Universidade do Vale do Rio dos Sinos e da Faculdade Cenecista de Bento Gonçalves.

$\longrightarrow$ doi:10.5335/rbceh.2012.023 


\section{Introdução}

O câncer de mama é o segundo tipo de câncer mais frequente no mundo e o mais comum entre as mulheres. (WHO, 2010). Segundo o Instituto Nacional de Câncer (Inca), o número de casos novos esperados para o Brasil em 2010 era de 49.240, com um risco estimado de 49 casos a cada 100 mil mulheres. No Rio Grande do Sul, estimava-se que 4.750 novos casos seriam registrados (81,57/100.000), sendo essa uma das mais altas taxas de incidência do país. (BRASIL, 2009).

No Brasil, o câncer de mama vem mostrando incidência e mortalidade ascendentes desde a década de 1960, a qual representou um marco social no país. A industrialização iniciada nas duas décadas anteriores passou a expressar seu reflexo na população: houve redução da taxa de natalidade, as mulheres começaram a inserir-se mais marcantemente no mercado de trabalho, a primeira gestação passou a ocorrer mais tardiamente, a urbanização alterou os hábitos alimentares da população e a esperança de vida ao nascer aumentou. Todos esses fatores, em maior ou menor grau, parecem guardar alguma relação com o câncer de mama. Com efeito, é nesse cenário de envelhecimento populacional e de maior exposição a fatores de risco que se insere o câncer de mama no Brasil. (KLIGERMAN, 1999).

O desenvolvimento de várias das formas mais comuns de câncer resulta de uma interação entre fatores endógenos e ambientais, sendo alguns dos mais notáveis a dieta, a prática de atividade física e a manutenção do peso corporal adequado. (WCRF, 2007).

A acentuada variação geográfica na incidência do câncer de mama no mundo foi uma das primeiras observações a sugerir que as diferenças nos hábitos alimentares poderiam estar relacionadas ao desenvolvimento dessa neoplasia. (TORRES-SÁNCHEZ et al., 2009). O efeito da migração adicionou mais informações nesse sentido, uma vez que mulheres japonesas que vivem no Japão, país que apresenta baixas taxas de incidência da doença e onde a dieta tradicional não é rica em lipídios, têm o risco de desenvolver câncer de mama aumentado ao emigrarem para o Havaí e mais ainda ao nascerem e permanecerem nos Estados Unidos. (NELSON, 2006).

Conhecer a distribuição dos fatores de risco para o câncer de mama em uma população é importante, pois possibilita ajudar na identificação dos grupos mais propensos a desenvolvê-lo. Para tanto, estratégias de educação em saúde poderiam ser adotadas no sentido de atuarem sobre os fatores relacionados ao estilo de vida desses indivíduos, os quais constituem hábitos potencialmente modificáveis ou controláveis, a fim de diminuir as taxas de incidência e mortalidade, além de atenuar o impacto psicossocial devido à doença. Do mesmo modo, programas de detecção precoce do câncer de mama poderiam ser criados, objetivando favorecer esses grupos de maior risco.

A Universidade da Terceira Idade (UNTI) é um programa institucional e extensionista da Universidade de Caxias do Sul (UCS), concebido para pessoas com 50 anos ou mais. A UNTI 
tem como objetivo conhecer o processo de envelhecimento do ser humano para contribuir na promoção do sujeito que envelhece e provocar transformações sociais na conquista de uma velhice bem-sucedida. Com a UNTI, pôde-se estender à comunidade o ensino e a pesquisa sobre o envelhecimento humano e todas as suas relações. (CORTELETTI; CASARA, 2007).

Assim contextualizado, o presente estudo tem por objetivo descrever a presença de fatores de risco associados ao desenvolvimento do câncer de mama em mulheres participantes da UNTI/UCS.

\section{Materiais e métodos}

Trata-se de um estudo transversal, descritivo e analítico. A amostra foi constituída por 45 mulheres, com idade entre 51 e 86 anos, sendo essa uma amostra por conveniência. Os dados foram coletados entre março e setembro de 2010.

Foram incluídas mulheres com idade igual ou superior a 50 anos, devidamente matriculadas e participantes da UNTI da UCS, sem história pessoal atual ou prévia de câncer de mama e que aceitaram fazer parte voluntariamente do estudo. A participação foi autorizada mediante assinatura do Termo de Consentimento Livre e Esclarecido (TCLE), e a pesquisa foi aprovada pelo Comitê de Ética em Pesquisa da Fundação Universidade de Caxias do Sul (CEP/FUCS), sob o parecer n. 355/10.

O presente estudo fez parte do projeto NUTENV, intitulado "Alimentação e envelhecimento: estudo do perfil nutricional, antropométrico e a relação com fatores socioeconômicos e culturais de idosos participantes da Universidade da Terceira Idade - UNTI", vinculado ao curso de Nutrição do Centro de Ciências da Saúde da UCS, aprovado pelo Comitê de Ética em Pesquisa da UCS, sob o protocolo n. 061/09.

As participantes foram convidadas a comparecer ao Laboratório de Avaliação Nutricional da UCS mediante agendamento telefônico. Com base em um questionário estruturado, foram obtidos dados socioeconômicos, reprodutivos, antropométricos, de estilo de vida e do consumo alimentar.

$\mathrm{O}$ instrumento de pesquisa deste estudo e os pontos de corte adotados como fatores de risco para o desenvolvimento do câncer de mama foram adaptados do Harvard Cancer Risk Index (COLDITZ et al., 2000) e dos questionários utilizados por Pinho e Coutinho (2007) e Di Pietro et al. (2007).

Considerou-se como fatores de risco relacionados ao desenvolvimento do câncer de mama a idade ( $\geq 50$ anos), a renda mensal (> 4 salários mínimos) e a escolaridade (ensino fundamental completo ou mais). Para os dados reprodutivos, foram considerados fatores de risco os seguintes pontos de corte: idade da menarca < 12 anos; nuliparidade; primeira gestação após os 30 anos; tempo de amamentação $<1$ ano, referente ao somatório de todos os filhos; idade da menopausa $\geq 55$ anos; tempo de uso de terapia de reposição hormonal $\geq 5$ anos; história familiar positiva de câncer de mama ou de outro tipo de câncer. Os fatores relacionados ao estilo de vida incluíram ausência de prática de exercício físico regular por 
pelo menos 3 vezes na semana, tabagismo e ex-tabagismo e consumo de mais de uma lata de cerveja, mais de uma taça de vinho e/ou mais de uma dose de bebidas destiladas/dia.

Os dados antropométricos foram obtidos pelas medidas de peso $(\mathrm{kg})$, estatura $(\mathrm{m})$ e da circunferência abdominal $(\mathrm{cm})$. O estado nutricional foi avaliado pelo índice de massa corporal (IMC) e classificado de acordo com os critérios da Organização Mundial da Saúde (OMS, 1995), a saber: $\mathrm{IMC} \leq 18,4 \mathrm{~kg} / \mathrm{m}^{2}$ para baixo peso, 18,5 a $24,9 \mathrm{~kg} / \mathrm{m}^{2}$ para eutrofia, 25 a $29,9 \mathrm{~kg} / \mathrm{m}^{2}$ para sobrepeso e IMC $\geq 30,0 \mathrm{~kg} / \mathrm{m}^{2}$ para obesidade, sendo esse último considerado fator de risco para o câncer de mama, assim como estatura $\geq$ $1,70 \mathrm{~m}$. Para a circunferência abdominal, definiu-se como fator de risco a medida $\geq$ $88 \mathrm{~cm}$, de acordo com o National Cholesterol Education Program (NCEP, 2002).

Para a aferição do peso, foi utilizada balança da marca Welmy ${ }^{\circledR}$, modelo 110 $\mathrm{CH}$, com capacidade mínima de $2 \mathrm{~kg}$ e máxima de $150 \mathrm{~kg}$ e divisão de $100 \mathrm{~g}$. As mulheres foram pesadas trajando roupas leves e sem sapatos. A altura foi medida utilizando-se um antropômetro vertical fixo à balança, estando a participante descalça, com os pés unidos, braços estendidos ao longo do corpo e olhar fixo na altura da linha do horizonte. (HEYWARD; STOLARCZYK, 2000). A medida da circunferência abdominal foi realizada conforme a I Diretriz brasileira de diagnóstico e tratamento da síndrome metabólica (2005), com a utilização de uma fita antropométrica inelástica, aplicada em posição horizontal, no ponto médio entre o rebordo costal inferior e a crista ilíaca. A avaliação foi obtida ao final de uma expiração normal.

O consumo alimentar foi caracterizado mediante a utilização de um Questionário de Frequência Alimentar (QFA) adaptado de Cardoso e Stocco (2000), Cardoso et al. (2001) e Furlan-Viebig e Pastor-Valero (2004). O QFA foi constituído de perguntas fechadas sobre a frequência de consumo alimentar (diário, semanal, mensal e anual) de 59 alimentos. Para o presente estudo, realizou-se a análise de 33 alimentos do QFA(considerando que para alguns existe a versão light), os quais se associam ao desenvolvimento do câncer de mama ou sugerem efeito protetor para a neoplasia.

As avaliações tiveram duração de no mínimo 40 e no máximo 90 minutos, tendo em vista que se empregou o questionário completo do projeto NUTENV. Todos os instrumentos de pesquisa foram aplicados por nutricionistas e/ou estudantes de nutrição, os quais eram bolsistas voluntários e/ou de iniciação científica, sendo todos previamente treinados.

Os dados obtidos foram plotados em planilha eletrônica do Microsoft Excel® versão 2007, e, posteriormente, foi realizada análise descritiva e analítica utilizando-se o software Statistical Package for the Social Sciences ${ }^{\circledR}$ (SPSS) versão 17.0. As variáveis quantitativas foram descritas através de média e desvio padrão (distribuição simétrica) ou mediana e amplitude de variação (distribuição assimétrica). As variáveis categóricas foram descritas por meio de frequências absolutas e relativas. Para avaliar a associação entre a classificação 
do IMC e os fatores de risco para câncer de mama, foi empregado o teste exato de Fisher. Para avaliar a associação entre a frequência de consumo dos alimentos e o número de fatores de risco com a classificação do IMC, o coeficiente de correlação de Spearman foi aplicado. No caso da associação da estatura com a idade da menarca, recorreu-se ao coeficiente de correlação de Pearson. O nível de significância estatística considerado foi de $5 \%(p \leq 0,05)$.

\section{Resultados}

No projeto NUTENV, foram entrevistadas inicialmente 50 mulheres, das quais 5 foram excluídas para o presente estudo, por haverem tido história pessoal atual ou prévia de câncer de mama. Dessa maneira, a amostra final constituiu-se de 45 participantes.

Em relação à caracterização da amostra, a idade média das mulheres entrevistadas foi de 64,3 $\pm 8,2$ anos. Quanto ao estado civil, $57,8 \%(\mathrm{n}=26)$ eram casadas e $28,9 \%(\mathrm{n}=13)$, viúvas. A renda mensal das participantes variou de 1 a 4 salários mínimos ou mais, registrando-se que $37,8 \%(\mathrm{n}=17)$ recebem de 2 a 3 salários mínimos e outras $37,8 \%$ $(\mathrm{n}=17)$ recebem mais do que 4 salários mínimos. Mais da metade das mulheres entrevistadas $(53,2 \%)$ tem como grau de escolaridade o ensino superior incompleto ou completo. As características socioeconômicas estão descritas na Tabela 1.
Tabela 1 - Características socioeconômicas da amostra.

\begin{tabular}{|c|c|}
\hline Variáveis & $\mathrm{n}=45$ \\
\hline $\begin{array}{l}\text { Idade (anos) - Média } \pm \text { DP } \\
\text { [Mínimo - Máximo] }\end{array}$ & $\begin{array}{r}64,3 \pm 8,2 \\
{[51-86]}\end{array}$ \\
\hline \multicolumn{2}{|l|}{ Estado civil - n (\%) } \\
\hline Solteira & $1(2,2)$ \\
\hline Casada & $26(57,8)$ \\
\hline Separada/Divorciada & $5(11,1)$ \\
\hline Viúva & $13(28,9)$ \\
\hline \multicolumn{2}{|l|}{$\begin{array}{l}\text { Renda mensal (salários } \\
\text { mínimos) - n (\%) }\end{array}$} \\
\hline 1 & $6(13,3)$ \\
\hline $2-3$ & $17(37,8)$ \\
\hline 4 & $5(11,1)$ \\
\hline$>4$ & $17(37,8)$ \\
\hline \multicolumn{2}{|l|}{ Escolaridade (anos) - n (\%) } \\
\hline Fundamental incompleto & $8(17,8)$ \\
\hline Fundamental completo & $5(11,1)$ \\
\hline Médio incompleto & $2(4,4)$ \\
\hline Médio completo & $6(13,3)$ \\
\hline Superior incompleto & $14(31,1)$ \\
\hline Superior completo & $10(22,2)$ \\
\hline
\end{tabular}

Quanto aos fatores reprodutivos, a idade média da menarca na amostra foi de 12,9 anos ( $D P=1,9)$. A primeira gestação ocorreu em média aos 23,6 anos de idade ( $\mathrm{DP}=3,7)$, sendo nulíparas apenas $4,4 \%(\mathrm{n}=2)$ das mulheres da amostra. Dentre as avaliadas, somente duas mulheres $(4,4 \%)$ ainda não haviam atingido a menopausa, e a idade média naquelas que já a haviam alcançado foi de 49,1 $\pm 5,4$ anos.

A análise dos dados antropométricos encontrada na Tabela 2 demonstra que a estatura média das participantes foi de $1,57 \mathrm{~m}$ ( $\mathrm{DP}=0,07)$. Quinze mulheres $(33,4 \%)$ foram classificadas como obesas segundo o IMC. A circunferência abdominal média foi de 99,3 cm ( $\mathrm{DP}=14,3$ ), e em $84,4 \%(n=38)$ das mulheres essa medida foi $\geq 88 \mathrm{~cm}$. 
Tabela 2 - Dados antropométricos.

\begin{tabular}{|c|c|}
\hline Variáveis & $\mathrm{n}=45$ \\
\hline Peso $(\mathrm{kg})$ - Média \pm DP [Mínimo - Máximo] & $70,8 \pm 14,1[49,7-112]$ \\
\hline Altura $(\mathrm{m})-$ Média \pm DP [Mínimo - Máximo] & $1,57 \pm 0,07[1,46-1,71]$ \\
\hline IMC atual $\left(\mathrm{kg} / \mathrm{m}^{2}\right)-$ Média \pm DP [Mínimo - Máximo] & $28,6 \pm 5,8[19,8-46,0]$ \\
\hline \multicolumn{2}{|l|}{ Classificação IMC - n (\%) } \\
\hline Eutrofia & $12(26,7)$ \\
\hline Sobrepeso & $18(40,0)$ \\
\hline Obesidade grau I & $9(20,0)$ \\
\hline Obesidade grau II & $3(6,7)$ \\
\hline Obesidade grau III & $3(6,7)$ \\
\hline $\begin{array}{l}\text { Circunferência abdominal }(\mathrm{cm})-\text { Média } \pm \text { DP [Mínimo - Máximo] } \\
>88 \mathrm{~cm}-n(\%)\end{array}$ & $\begin{array}{r}99,3 \pm 14,3[80,0-147,0] \\
38(84,4)\end{array}$ \\
\hline
\end{tabular}

$\mathrm{n}=$ número de participantes; $\mathrm{DP}=$ desvio padrão $\%$ = porcentagem $; \mathrm{kg}=$ quilogramas $; \mathrm{m}=$ metros $; \mathrm{IMC}=$ índice de massa corporal; $\mathrm{kg} / \mathrm{m}^{2}=$ quilogramas por metro quadrado; $\mathrm{cm}=$ centímetros.

\section{A Tabela 3 apresenta a avaliação dos de acordo com os pontos de corte previa- fatores de risco para o câncer de mama mente adotados.}

Tabela 3 - Avaliação dos fatores de risco para câncer de mama.

\begin{tabular}{lr}
\hline \multicolumn{1}{c}{ Variáveis } & $\mathrm{n}=45 \mathrm{n}(\%)$ \\
\hline Idade $\geq 50$ anos & $45(100)$ \\
Renda mensal > 4 salários mínimos & $17(37,7)$ \\
Escolaridade (ensino fundamental completo ou mais) & $37(82,2)$ \\
Idade da menarca < 12 anos & $12(26,7)$ \\
Nuliparidade & $2(4,4)$ \\
Idade na 1 a gestação > 30 anos & $2(4,4)$ \\
Não ter amamentado uma criança & $4(8,9)$ \\
Amamentação < 1 ano* & $28(62,2)$ \\
Idade na menopausa $\geq 55$ anos & $7(15,6)$ \\
Uso de terapia de reposição hormonal & $13(28,9)$ \\
Tempo de reposição hormonal $\geq 5$ anos** & $9 / 13(69,2)$ \\
História familiar positiva para câncer de mama & $7(15,6)$ \\
História familiar positiva para câncer em geral & $30(66,7)$ \\
Estatura $\geq 1,70 m$ & $1(2,2)$ \\
Obesidade & $15(33,3)$ \\
Ausência de prática de atividade física*** & $9(20,0)$ \\
Fumante & $2(4,4)$ \\
Ex-fumante & $13(28,9)$ \\
Ingestão de álcool*** & $20(44,4)$ \\
Cerveja > 350 ml (1 lata/dia) & $0(0,0)$ \\
Uísque > 40 ml (1 dose/dia) & $0(0,0)$ \\
Vinho > 150 ml (1 taça/dia) & $3 / 17(17,6)$ \\
Cachaça > 40 ml (1 dose/dia) & $0(0,0)$ \\
No de fatores de risco - Média \pm DP [Mínimo - Máximo] & $5,0 \pm 1,9[1,0-10,0]$ \\
\hline
\end{tabular}

$\mathrm{n}=$ número de participantes; $\%=$ porcentagem; $\mathrm{DP}=$ desvio padrão $\mathrm{m}=$ metros; $\mathrm{ml}=$ mililitros.

* O tempo de amamentação foi, em mediana, de 8 meses com mínimo de 1 mês e máximo de 7 anos.

** O tempo de reposição hormonal foi, em mediana, de 5 anos com mínimo de 2 e máximo de 25 anos.

*** $36(80,0 \%)$ praticam atividade física. Destas, $12(33,3 \%)$ fazem pilates/alongamento, $10(27,8 \%)$ praticam caminhada, 7 (19,4\%) fazem hidroginástica/natação, 7 (19,4\%) fazem dança, 7 (19,4\%) praticam aeróbica, $5(13,9 \%)$ frequentam a academia/ fazem musculação, $5(13,9 \%)$ praticam yoga e $1(2,8 \%)$ pratica vôlei. A duração média é de $1,2 \mathrm{~h} \pm 0,5 \mathrm{~h}[0,7-2,0]$ com frequência semanal de 1 a $2 \mathrm{x}(\mathrm{n}=25 ; 69,4 \%), 3$ a 4x $(\mathrm{n}$ $=6 ; 16,7 \%)$ e $>4 x(\mathrm{n}=5 ; 13,9 \%)$.

***** Destes, 17 (85\%) ingerem vinho; 5 (25\%), cachaça; 4 (20\%), cerveja; e 1 (5\%), uísque. A mediana de consumo dessas bebidas foi de, respectivamente, $28 \mathrm{ml}$ [4 - 243], $14 \mathrm{ml}$ [8 - 17], $36 \mathrm{ml}$ [11 - 86] e $10 \mathrm{ml}$. 
Quanto aos fatores de risco para câncer de mama, houve uma associação direta estatisticamente significante entre a classificação do IMC e o número de fatores de risco para câncer de mama $\left(\mathrm{r}_{\mathrm{s}}=0,537 ; \mathrm{p}<0,001\right)$. Mulheres mais obesas apresentam significativamente mais fatores de risco, em média sete, o que representa dois a mais do que a média do grupo - sendo um já esperado, ou seja, a própria obesidade. Nenhum fator de risco associou-se significativamente com a obesidade, mas três deles apresentaram um valor $\mathrm{p}<0,20$ e merecem ser citados devido ao pequeno tamanho da amostra. Mulheres obesas revelaram-se mais sedentárias, mais fumantes e ex-fumantes do que as não obesas, mas não de forma estatisticamente significante. Os percentuais encontrados para as variáveis citadas estão sistematizados na Tabela 4.

Tabela 4 - Associação entre estado nutricional atual e fatores de risco para câncer de mama.

\begin{tabular}{|c|c|c|c|}
\hline \multirow[t]{2}{*}{$\begin{array}{l}\text { Fatores de } \\
\quad \text { risco }\end{array}$} & $\begin{array}{l}\text { Obesas } \\
(n=15)\end{array}$ & $\begin{array}{c}\text { Não } \\
\text { obesas } \\
(n=30)\end{array}$ & \multirow[t]{2}{*}{$\mathrm{p}^{*}$} \\
\hline & n (\%) & n (\%) & \\
\hline \multicolumn{4}{|l|}{ Fumante } \\
\hline Sim & $2(13,3)$ & $0(0,0)$ & 0,106 \\
\hline Não & $13(86,7)$ & $30(100,0)$ & \\
\hline \multicolumn{4}{|l|}{ Ex-fumante } \\
\hline Sim & $7(46,7)$ & $6(20,0)$ & 0,086 \\
\hline Não & $8(53,3)$ & $24(80,0)$ & \\
\hline \multicolumn{4}{|l|}{$\begin{array}{l}\text { Prática de } \\
\text { atividade } \\
\text { física }\end{array}$} \\
\hline Sim & $10(66,7)$ & $26(86,7)$ & 0,135 \\
\hline Não & $5(33,3)$ & $4(13,3)$ & \\
\hline
\end{tabular}

A correlação de dados realizada por meio de análise estatística demonstrou que não houve associação estatisticamente significante entre a estatura da mulher e a idade da menarca $(r=0,098$; $\mathrm{p}=0,522$ ).

Os resultados em relação ao consumo alimentar, obtidos por meio do QFA, encontram-se descritos na Tabela 5. A análise evidencia que mais da metade das entrevistadas $(51,1 \%)$ ingere produtos integrais diariamente. Vinte e oito mulheres $(62,2 \%)$ consomem feijão com frequência semanal; a maior parte da amostra $(88,9 \%)$ come frutas todos os dias, sendo a banana a mais ingerida ( $\mathrm{n}$ = 27); mais da metade das investigadas $(57,8 \%)$ consome vegetais crus todos os dias, sendo a alface o mais frequente $(55,6 \%)$; e a carne vermelha é consumida pela maioria das mulheres da amostra $(71,1 \%)$ semanalmente. Em relação ao grupo alimentar do leite e derivados, o leite desnatado é o mais consumido diariamente $(35,6 \%)$; o leite integral e o semidesnatado foram relatados como nunca ingeridos por $71,1 \%(\mathrm{n}=32) \mathrm{e}$ $84,4 \%(\mathrm{n}=34)$ da amostra, respectivamente. O queijo mais consumido foi o do tipo mussarela, registrando-se que $31,1 \%$ ( $\mathrm{n}=14$ ) das mulheres o consomem diariamente. No que se refere ao grupo dos óleos e das gorduras, os óleos vegetais $(91,1 \%)$ foram relatados como os mais consumidos todos os dias. A maior parte da amostra não tem o hábito de ingerir manteiga, margarina, maionese e gordura animal, além de não consumir produtos light. 
Tabela 5 - Avaliação do Questionário de Frequência Alimentar (QFA).

\begin{tabular}{|c|c|c|c|c|c|}
\hline \multirow{2}{*}{ Alimentos } & Diário & Semanal & Mensal & Anual & Nunca \\
\hline & $\mathrm{n}(\%)$ & $\mathrm{n}(\%)$ & $\mathrm{n}(\%)$ & $\mathrm{n}(\%)$ & $\mathrm{n}(\%)$ \\
\hline Pão integral & $20(44,4)$ & $15(33,3)$ & $3(6,7)$ & $1(2,2)$ & $6(13,3)$ \\
\hline Produtos integrais & $23(51,1)$ & $12(26,7)$ & $5(11,1)$ & $0(0,0)$ & $5(11,1)$ \\
\hline Grãos & $14(31,1)$ & $25(55,6)$ & $6(13,3)$ & $0(0,0)$ & $0(0,0)$ \\
\hline Feijão & $9(20,0)$ & $28(62,2)$ & $8(17,8)$ & $0(0,0)$ & $0(0,0)$ \\
\hline Lentilha & $2(4,4)$ & $16(35,6)$ & $15(33,3)$ & $6(13,3)$ & $6(13,3)$ \\
\hline Soja & $0(0,0)$ & $3(6,7)$ & $3(6,7)$ & $1(2,2)$ & $38(84,4)$ \\
\hline Grão-de-bico & $0(0,0)$ & $0(0,0)$ & $9(20,0)$ & $4(8,9)$ & $32(71,1)$ \\
\hline Frutas & $40(88,9)$ & $5(11,1)$ & $0(0,0)$ & $0(0,0)$ & $0(0,0)$ \\
\hline Banana & $27(60,0)$ & $15(33,3)$ & $3(6,7)$ & $0(0,0)$ & $0(0,0)$ \\
\hline Abacaxi & $1(2,2)$ & $11(24,4)$ & $22(48,9)$ & $4(8,9)$ & $7(15,6)$ \\
\hline Laranja & $13(28,9)$ & $21(46,7)$ & $7(15,6)$ & $1(2,2)$ & $3(6,7)$ \\
\hline Mamão & $22(48,9)$ & $18(40,0)$ & $4(8,9)$ & $0(0,0)$ & $1(2,2)$ \\
\hline Uva & $14(31,1)$ & $14(31,1)$ & $6(13,3)$ & $7(15,6)$ & $4(8,9)$ \\
\hline Vegetais crus & $26(57,8)$ & $10(22,2)$ & $3(6,7)$ & $1(2,2)$ & $5(11,1)$ \\
\hline Vegetais cozidos & $16(35,6)$ & $26(57,8)$ & $2(4,4)$ & $0(0,0)$ & $1(2,2)$ \\
\hline Alface & $25(55,6)$ & $15(33,3)$ & $3(6,7)$ & $0(0,0)$ & $2(4,4)$ \\
\hline Beterraba & $1(2,2)$ & $22(48,9)$ & $11(24,4)$ & $0(0,0)$ & $11(24,4)$ \\
\hline Cenoura & $7(15,6)$ & $30(66,7)$ & $4(8,9)$ & $0(0,0)$ & $4(8,9)$ \\
\hline Tomate & $13(28,9)$ & $27(60,0)$ & $4(8,9)$ & $0(0,0)$ & $1(2,2)$ \\
\hline Batata-inglesa & $2(4,4)$ & $35(77,8)$ & $4(8,9)$ & $0(0,0)$ & $4(8,9)$ \\
\hline Carne vermelha & $6(13,3)$ & $32(71,1)$ & $6(13,3)$ & $0(0,0)$ & $1(2,2)$ \\
\hline Creme de leite normal & $0(0,0)$ & $9(20,0)$ & $13(28,9)$ & $3(6,7)$ & $20(44,4)$ \\
\hline Creme de leite light & $1(2,2)$ & $3(6,7)$ & $2(4,4)$ & $0(0,0)$ & $39(86,7)$ \\
\hline Requeijão normal & $2(4,4)$ & $4(8,9)$ & $8(17,8)$ & $1(2,2)$ & $30(66,7)$ \\
\hline Requeijão light & $3(6,7)$ & $6(13,3)$ & $1(2,2)$ & $1(2,2)$ & $34(75,6)$ \\
\hline Leite integral & $12(26,7)$ & $0(0,0)$ & $1(2,2)$ & $0(0,0)$ & $32(71,1)$ \\
\hline Leite semidesnatado & $6(13,3)$ & $0(0,0)$ & $1(2,2)$ & $0(0,0)$ & $38(84,4)$ \\
\hline Leite desnatado & $16(35,6)$ & $0(0,0)$ & $1(2,2)$ & $0(0,0)$ & $28(62,2)$ \\
\hline Queijo mussarela & $14(31,1)$ & $16(35,6)$ & $2(4,4)$ & $0(0,0)$ & $13(28,9)$ \\
\hline Queijo lanche & $4(8,9)$ & $2(4,4)$ & $1(2,2)$ & $0(0,0)$ & $38(84,4)$ \\
\hline Queijo colonial & $4(8,9)$ & $5(11,1)$ & $0(0,0)$ & $0(0,0)$ & $36(80,0)$ \\
\hline Manteiga & $6(13,3)$ & $5(11,1)$ & $2(4,4)$ & $2(4,4)$ & $30(66,7)$ \\
\hline Margarina & $7(15,6)$ & $10(22,2)$ & $4(8,9)$ & $2(4,4)$ & $22(48,9)$ \\
\hline Bacon & $0(0,0)$ & $2(4,4)$ & $11(24,4)$ & $5(11,1)$ & $27(60,0)$ \\
\hline Maionese normal & $0(0,0)$ & $7(15,6)$ & $8(17,8)$ & $1(2,2)$ & $29(64,4)$ \\
\hline Maionese light & $0(0,0)$ & $2(4,4)$ & $2(4,4)$ & $1(2,2)$ & $40(88,9)$ \\
\hline Óleos vegetais & $41(91,1)$ & $1(2,2)$ & $1(2,2)$ & $0(0,0)$ & $2(4,4)$ \\
\hline Óleos animais & $2(4,4)$ & $1(2,2)$ & $1(2,2)$ & $0(0,0)$ & $41(91,1)$ \\
\hline Sorvete normal & $0(0,0)$ & $14(31,1)$ & $17(37,8)$ & $6(13,3)$ & $8(17,8)$ \\
\hline Sorvete light & $0(0,0)$ & $1(2,2)$ & $0(0,0)$ & $0(0,0)$ & $44(97,8)$ \\
\hline Molho pronto & $0(0,0)$ & $6(13,3)$ & $5(11,1)$ & $1(2,2)$ & $33(73,3)$ \\
\hline
\end{tabular}

$\mathrm{n}=$ número de participantes; $\%=$ porcentagem. 
De acordo com os dados obtidos, houve associação inversa estatisticamente significante entre a ingestão de alface $\left(\mathrm{r}_{\mathrm{s}}=-0,295 ; \mathrm{p}=0,050\right)$, beterraba $\left(\mathrm{r}_{\mathrm{s}}=\right.$ $-0,302 ; p=0,043)$ e queijo, independentemente do tipo $\left(r_{s}=-0,369 ; p=0,013\right)$, e a classificação do IMC. Constatou-se que mulheres mais obesas tendem a comer menos frequentemente esses alimentos.

\section{Discussão}

O câncer de mama é uma das neoplasias mais estudadas no mundo inteiro, mas ainda existem muitas pendências e muitas controvérsias sobre os seus determinantes. A maioria dos estudos enfatiza a questão das variáveis associadas em grupos que já desenvolveram a doença, e pouco se sabe sobre a prevalência desses fatores em mulheres assintomáticas. (PINHO; COUTINHO, 2007).

Além do sexo, a idade elevada é um dos fatores de risco mais bem estabelecidos na associação com o câncer de mama, pois provavelmente reflete os danos ao DNA acumulados durante a vida. Embora esse tipo de câncer possa ocorrer em idades menores, trata-se de uma doença, em geral, relacionada ao envelhecimento, visto que cerca de $75 \%$ dos casos ocorrem após os 50 anos. (NBOCC, 2009). Neste trabalho, todas as mulheres da amostra encontram-se na idade de maior risco para o desenvolvimento da doença, mas é válido lembrar que a UNTI oferece atividades para maiores de 50 anos.

Os dados deste estudo mostram um grupo composto, em sua maioria, por mulheres com elevada renda e escolaridade. De acordo com Tessaro (2001), a neoplasia de mama é uma doença que ocorre mais entre as mulheres de maior nível socioeconômico, provavelmente refletindo fatores ligados ao estilo de vida, em que a elevada escolaridade e renda resultam em idade tardia para ter o primeiro filho, por exemplo. Por outro lado, o estudo realizado por $\mathrm{Di}$ Pietro et al. (2007) indicou que o risco de câncer de mama foi maior entre as mulheres de baixa renda e menor nível de escolaridade, o que, segundo os autores, pode estar associado a um estilo de vida mais estressante, dieta inadequada e dificuldade de acesso aos serviços de saúde e informações sobre a prevenção da doença.

Ao contrário das variáveis socioeconômicas, os fatores hormonais e reprodutivos são mais bem estabelecidos na etiologia do câncer de mama. A menarca precoce e a menopausa tardia são consideradas fatores de risco, devido à maior exposição aos estímulos do estrogênio durante a vida. (NBOCC, 2009). A idade média da menarca (12,9 anos) nas mulheres deste trabalho foi semelhante à encontrada (13 anos) por Matos et al. (2010), em trabalho realizado no estado do Paraná, e por Lima et al. (2001) (12,3 anos) em estudo com mulheres indígenas no Mato Grosso do Sul. A idade média da menopausa (49,1 anos), por sua vez, também se assemelha à evidenciada por Matos et al. (2010), tendo a maior parte das mulheres entrevistadas atingido a menopausa entre 48 e 55 anos.

A prevalência de mulheres que amamentaram por menos de um ano $(62,2 \%)$ foi maior que a encontrada $(36,4 \%)$ por Pinho e Coutinho (2007), em estudo realizado no Rio de Janeiro com usuárias 
de uma Unidade Básica de Saúde com idade entre 25 e 88 anos. Conforme o Painel do World Cancer Research Fund (WCRF, 2007), a lactação está associada a um aumento da diferenciação das células mamárias e à menor exposição aos hormônios estrogênicos durante a amenorreia que acompanha a lactação. Além disso, a forte esfoliação do tecido mamário durante esse período e a apoptose maciça de células epiteliais ao final da amamentação poderiam diminuir o risco pela eliminação de células com danos ao DNA. O painel foi o primeiro relatório importante relacionado à prevenção de câncer a fazer uma recomendação especificamente para amamentação, visando à prevenção do câncer de mama.

No presente estudo, $71,1 \%$ das mulheres nunca fizeram uso de terapia de reposição hormonal, prevalência ligeiramente maior que a encontrada $(69,2 \%)$ por Matos et al. (2010). Borghesan et al. (2008), por sua vez, observaram que $81,2 \%$ da sua amostra nunca havia feito uso desse tipo de tratamento. Acredita-se que o efeito da terapia de reposição hormonal não seja indutor do câncer de mama, mas provavelmente promotor, ou seja, aumentaria o crescimento de tumores pré-existentes, explicando o acréscimo de cânceres associado a seu uso. (MAJLIS, 2008).

Outra característica importante observada na nossa amostra foi que $80 \%$ das mulheres avaliadas praticam exercícios físicos; porém, destas, somente $16,7 \%$ os fazem com regularidade $\geq 3$ vezes por semana. Caminhada e alongamentos foram as atividades mais praticadas. No estudo de Pinho e Coutinho (2007), mais da metade das mulheres da amostra era sedentária, e, dentre as que praticavam exercícios físicos regulares, a caminhada também foi o mais frequente. Silva et al. (2009), ao explorarem a presença de fatores de risco para câncer de mama em mulheres indígenas no Paraná, verificou que apenas $17,3 \%$ praticavam exercícios pelo menos três vezes na semana regularmente. A atividade física parece exercer efeitos nos níveis hormonais e, dessa maneira, pode diminuir o risco de câncer de mama. (ROMIEU; LAJOUS, 2009). Nesse contexto, é válido lembrar, ainda, que a UNTI oferece diversas disciplinas relacionadas à prática de atividade física, embora a maior parte da amostra se exercite com frequência $<3$ vezes na semana.

Um hábito positivo encontrado entre as mulheres entrevistadas é que somente 4,4\% delas são tabagistas; entretanto, é importante destacar o fato de que essas mesmas mulheres foram classificadas como obesas segundo o IMC. No estudo de Lima et al. (2001), o tabagismo foi referido por apenas $7 \%$ das mulheres da amostra. A revisão do National Breast and Ovarian Cancer Centre (NBOCC, 2009) sobre fatores de risco para câncer de mama destaca que a relação entre o tabagismo e esse tipo de câncer ainda é complicada, pois não existem evidências consistentes acerca dessa interação. Ainda assim, é recomendação do Ministério da Saúde, conforme o Documento de consenso para controle do câncer de mama (2004), que alguns fatores de risco, especialmente a obesidade e o tabagismo, sejam alvo de ações visando à promoção à saúde.

O álcool é o fator dietético para o qual a associação com o câncer de mama 
é mais consistente. (ROMIEU; LAJOUS, 2009). As bebidas alcoólicas podem conter substâncias carcinogênicas (acetaldeído), sem contar que o álcool, por si só, já é capaz de solubilizar outros carcinógenos externos para que penetrem na célula. (SÁNCHEZ et al., 2009). No presente estudo, atentou-se para o fato de que $17,6 \%$ das mulheres que consomem vinho todos os dias o fazem em uma quantidade maior que a recomendada para a prevenção do câncer de mama, porém não de maneira demasiada. Quanto a isso, é importante registrar que tal fato pode estar vinculado à cultura italiana local, bem como à adesão à bebida para controle de doença cardiovascular, função que lhe é atribuída popularmente.

No presente trabalho, a estatura não foi associada de maneira significante com a idade da menarca. O estudo de caso-controle realizado por Vasconcelos et al. (2001), no Rio de Janeiro, demonstrou que as mulheres na pré-menopausa eram mais altas e tiveram a menarca mais cedo do que as mulheres pós-menopáusicas. De acordo com a revisão feita por Friedenreich (2001) sobre fatores antropométricos e câncer de mama, o risco de desenvolver a neoplasia aumenta em mulheres mais altas, tanto na pré quanto na pós-menopausa.

A obesidade, por seu turno, mostrou-se presente em $33,4 \%$ das mulheres, prevalência um pouco maior que a encontrada por Pinho e Coutinho (2007), em que $30 \%$ de sua amostra apresentaram IMC $\geq 30 \mathrm{~kg} / \mathrm{m}^{2}$. Amaral et al. (2010), em estudo conduzido em Portugal com 71 mulheres com idade média de 60 anos, portadoras de câncer de mama, observa- ram que $34 \%$ delas foram classificadas como obesas segundo indicador de IMC. Esse fator de risco parece apresentar uma interação com o status menopausal, pois mulheres acima do peso na pré-menopausa correm um risco mais baixo, enquanto mulheres pós-menopáusicas com excesso de peso têm maior risco de desenvolver câncer de mama. (ROMIEU; LAJOUS, 2009). A gordura corporal afeta diretamente os níveis de muitos hormônios circulantes, como insulina, fatores de crescimento semelhantes à insulina e estrogênios, criando um ambiente que encoraja a carcinogênese e desencoraja a apoptose. (WCRF, 2007). Além disso, destaca-se que as mulheres obesas deste estudo apresentaram mais fatores de risco para o câncer de mama, de maneira estatisticamente significante. Assim sendo, a atuação nos fatores que são passíveis de modificação merece ser considerada.

Embora a gordura corporal já seja causa convincente de câncer de mama, sobretudo em mulheres na pós-menopausa, ainda não há evidências consistentes acerca, especificamente, da gordura abdominal. Ainda assim, o relatório do WCRF (2007) sugere que essa gordura seja, provavelmente, uma causa do câncer de mama, por associar-se ao aumento dos níveis de estrogênio circulante e por promover a diminuição da sensibilidade à insulina. No presente estudo, considerou-se como aumento da gordura abdominal a circunferência do abdômen $\geq 88 \mathrm{~cm}$, assim como sugerido pelo NCEP (2002), tendo em vista que pouco se sabe sobre esse parâmetro especificamente para câncer de mama, 
e observou-se que $84,4 \%$ das mulheres da amostra apresentaram essa medida aumentada. No estudo de Amaral et al. (2010), 62\% das pacientes avaliadas tiveram uma circunferência $\geq 88 \mathrm{~cm}$. Em relação a essa variável antropométrica, é válido lembrar que, com o envelhecimento, somada ao aumento da gordura corporal, observa-se redistribuição desse tecido, havendo diminuição nos membros e acúmulo preferencialmente na região abdominal. (PERISSINOTTO et al., 2002).

De maneira geral, a alimentação das mulheres da amostra, em seu conjunto, mostrou-se adequada, especialmente no que diz respeito ao consumo diário de frutas, verduras e produtos integrais e à baixa ingestão de alimentos fonte de gordura. O estudo de caso-controle conduzido por Lima et al. (2008) no estado da Paraíba, Nordeste do Brasil, identificou que o consumo de frutas e sucos, feijão e leite e derivados apresentou uma forte associação com a redução do risco de câncer de mama.

Alguns achados sugerem que práticas alimentares como o vegetarianismo podem estar associadas à diminuição do risco de câncer de mama, devido à sua relação com um maior consumo de frutas e verduras. (SILVA et al., 2002). Os vegetais contêm em sua composição moléculas antioxidantes capazes de prevenir a formação e a proliferação de tumores (WEINER et al., 2010), enquanto as fibras presentes nesses alimentos podem desempenhar um papel no risco do câncer de mama, diminuindo a reabsorção intestinal de estrogênio e, portanto, atenuando seus níveis circulantes.
(COHEN, 1999). No presente estudo, a análise demonstrou que houve associação inversa estatisticamente significante entre a ingestão de alface e beterraba e a classificação do IMC. Mulheres obesas tendem a comer menos frequentemente esses vegetais, os quais são fonte de micronutrientes e fibras, prováveis fatores de proteção para o câncer de mama.

Cibeira e Guaragna (2006), em estudo de revisão sobre o lipídio dietético como fator de risco para o câncer de mama, apontaram que existem evidências de que a dieta rica em ácido graxo linoleico ( $\omega-6)$ estimularia vários estágios no desenvolvimento do câncer mamário. Por outro lado, o ácido graxo $\omega-3$ parece prevenir o câncer pela influência sobre a atividade de enzimas e proteínas relacionadas à proliferação celular. No presente estudo, a maior parte da amostra $(91,1 \%)$ consome óleos vegetais diariamente; entretanto, é importante ressaltar que o QFA empregado não discriminou seu tipo e sua quantidade. Amaral et al. (2010) observaram que 92\% de sua amostra apresentaram uma baixa ingestão semanal de óleos de soja e girassol, além de um elevado consumo (80\%) de azeite de oliva.

Ainda de acordo com Amaral et al. (2010), uma ingestão elevada de gordura saturada poderia aumentar os níveis circulantes de estrogênio, os quais são capazes de promover o crescimento de células mamárias malignas. Os autores verificaram que $48 \%$ da sua amostra apresentaram um alto consumo de manteiga, alimento fonte de gordura saturada, ao passo que no presente estudo $66,7 \%$ das mulheres nunca ingerem 
esse produto. Todavia, apesar dos vários trabalhos que têm procurado associar a gordura da dieta e o câncer de mama, nenhuma conclusão pode ser firmada até o momento, pois os processos biológicos que levariam à carcinogênese ainda não estão bem elucidados. (ROMIEU; LAJOUS, 2009).

Lima et al. (2008) evidenciaram, igualmente, que o consumo de carnes vermelhas esteve positivamente associado ao risco de câncer de mama. Di Pietro et al. (2007), em estudo de caso-controle realizado em Joinvile, Santa Catarina, também demonstraram que a ingestão de carne vermelha foi significativamente associada ao risco da doença. Esse alimento, cozido em altas temperaturas, contém substâncias carcinogênicas potentes, tais como aminas heterocíclicas e outros compostos nitrogenados que se acredita aumentarem o risco de câncer de mama (WCRF, 2007). No presente estudo, observa-se que a carne vermelha é consumida, pela maioria das mulheres, com frequência semanal, sendo tal consumo provavelmente moderado, fato positivo para a prevenção da doença. Todavia, a quantificação de sua ingestão deverá ser melhor investigada.

Nessa perspectiva, cabe observar que o QFA apresenta algumas limitações, tais como a dependência da memória dos entrevistados sobre hábitos do passado. Aspectos como idade, grau de escolaridade e origem étnica também podem interferir na validade das informações coletadas. (FISBERG et al., 2005). Além disso, no presente estudo, realizou-se a análise apenas da frequência de tempo dos alimentos consumidos, não tendo sido considerados tamanho e quantidade de porções.

Embora o papel da dieta na causa do câncer de mama ainda não se encontre totalmente estabelecido, o Painel do WCRF (2007) preconiza como medidas preventivas a restrição do consumo de álcool e de carnes vermelhas, especialmente as processadas e ricas em gordura, bem como preferência por grãos integrais a grãos refinados e açúcares.

\section{Conclusão}

As evidências demonstram que os fatores de risco para o desenvolvimento do câncer de mama parecem relacionar-se entre si, o que torna difícil avaliar a real contribuição de um fator isolado na gênese da doença. A maior parte dessas variáveis constitui-se, ainda, de fatores individuais que não podem ser modificados.

$\mathrm{O}$ presente estudo apresenta algumas limitações, destacando-se o número reduzido da amostra, devido à dificuldade de recrutamento das participantes, as quais deveriam comparecer a um determinado local em horário previamente agendado para a aplicação do questionário. Além disso, as mulheres da UNTI têm um elevado nível sociocultural e encontram-se em um meio que permite fácil acesso às mais variadas informações, sobretudo àquelas relacionadas a um estilo de vida saudável, o que pode refletir nos bons hábitos relatados quanto ao consumo alimentar, de maneira geral.

Apesar dessas limitações, os resultados obtidos chamam a atenção para a alta prevalência de sobrepeso 
e obesidade observada na amostra e, ainda, para o fato de que as mulheres obesas mostraram-se mais sedentárias, fumantes e ex-fumantes. Esses fatores são importantes para a promoção de estratégias de educação em saúde, as quais devem incluir intervenções de educação e orientação nutricional, por serem características que podem ser alteradas em nível individual. Assim, essas ações educativas poderiam atuar na prevenção do câncer de mama, ou, pelo menos, na sua atenuação, bem como no controle de outras doenças.

Risk factors associated with breast cancer in a sample of members of a university for seniors

\section{Abstract}

Breast cancer is the most common type of cancer among women, and is considered a disease of extreme importance to public health worldwide. It is believed that the rising incidence and mortality are related to higher exposure to risk factors and to population aging. The aim of this study was to describe the presence of risk factors associated with breast cancer in women at the University for Seniors of the University of Caxias do Sul, RS. The study was a crosssectional, descriptive and analytical type, with a sample of 45 women, aged between 51 and 86 years. The data collection was made in the period of March up to September 2010. It was collected socioeconomic, reproductive, anthropometric, lifestyle and food intake data. The participants showed a profile of high income and education. The average age of the menarche was 12.9 \pm 1.9 years and the menopause $49.1 \pm 5.4$ years; $62.2 \%$ have breastfed for less than one year, $33.4 \%$ were classified as obese according to $\mathrm{BMI}, 17.6 \%$ exercised regularly. The obese women presented significantly more risk factors than the others.
Generally speaking the food showed to be adequate, particularly in daily consumption of fruits, vegetables and whole food, and low fat intake. Nevertheless, the study draws attention to the high prevalence of overweight and obesity in the sample, factors that can be changed in an individual level through education interference and nutritional orientation.

Keywords: Aging. Breast neoplasms. Risk factors. Life style. Diet.

\section{Agradecimentos}

Às idosas que participaram voluntariamente desta pesquisa, à UNTI e à sua coordenação e, em especial, às bolsistas vinculadas ao Projeto NUTENV Caroline Demori e Juliana Caprini.

\section{Referências}

AMARAL, P. et al. Body fat and poor diet in breast cancer women. Nutrición Hospitalaria, Madrid, v. 25, n. 3, p. 456-461, 2010.

BORGHESAN, D. H. et al. Câncer de mama e fatores associados. Ciência, Cuidado e Saúde, Maringá, v. 7, supl. 1, p. 62-68, 2008.

BRASIL. Ministério da Saúde. Controle do câncer de mama: documento de consenso. Rio de Janeiro: Ministério da Saúde/Instituto Nacional de Câncer, 2004. . Estimativa 2010: inci-

dência de câncer no Brasil. Rio de Janeiro: Ministério da Saúde/Instituto Nacional de Câncer, 2009.

CARDOSO, M. A.; STOCCO, P. R. Desenvolvimento de questionário quantitativo de frequência alimentar em imigrantes japoneses de São Paulo. Cadernos de Saúde Pública. Rio de Janeiro, v. 16, n. 1, p. 107-114, 2000.

CARDOSO, M. A. et al. Reproducibility and relative validity of a food frequency questionnaire among women of japanese ancestry 
living in Brazil. Nutrition Research, Amsterdam, v. 21, n. 5, p. 725-733, 2001.

CIBEIRA, G. H.; GUARAGNA, R. M. Lipídio: fator de risco e prevenção do câncer de mama. Revista de Nutrição, Campinas, v. 19, n. 1, p. 65-75, 2006.

COHEN, L. A. Dietary fiber and breast cancer. Anticancer Research, Kapandriti, v. 19, supl. 5A, p. 3685-3688, 1999.

COLDITZ, G. A. et al. Harvard report on cancer prevention volume 4: Harvard Cancer Risk Index. Cancer Causes and Control, Heidelberg, v. 11, n. 6, p. 477-488, 2000.

CORTELETTI, I. A.; CASARA, M. B. Projeto pedagógico: Universidade da Terceira Idade UNTI. Caxias do Sul, RS: Educs, 2007.

DI PIETRO, P. F. et al. Breast cancer in Southern Brazil: association with past dietary intake. Nutrición Hospitalaria, Madrid, v. 22, n. 5, p. 565-572, 2007.

FISBERG, R. M. et al. Métodos de inquéritos alimentares. In: FISBERG, R. M. et al. (Org.). Inquéritos alimentares: métodos e bases científicos. São Paulo: Manole, 2005. p. 1-31.

FRIEDENREICH, C. M. Review of anthropometric factors and breastcancer risk. European Journal of Cancer Prevention, Hasselt, v. 10, p. 15-32, 2001.

FURLAN-VIEBIG, R.; PASTOR-VALERO, M. Desenvolvimento de um questionário de frequência alimentar para o estudo de dieta e doenças não transmissíveis. Revista de Saúde Pública, São Paulo, v. 38, n. 4, p. 581-584, 2004.

HEYWARD, V. H.; STOLARCZYK, L. M. Avaliação da composição corporal aplicada. São Paulo: Manole, 2000.

I DIRETRIZ brasileira de diagnóstico e tratamento da síndrome metabólica. Arquivos Brasileiros de Cardiologia, Rio de Janeiro, v. 84, supl. 1, p. 1-28, 2005.

KLIGERMAN, J. O câncer como um indicador de saúde no Brasil (Editorial). Revista
Brasileira de Cancerologia, Rio de Janeiro, v. 45, n. 3, 1999.

LIMA, F. E. L. et al. Diet and cancer in Northeast Brazil: evaluation of eating habits and food group consumption in relation to breast cancer. Cadernos de Saúde Pública, Rio de Janeiro, v. 24, n. 4, p. 820-828, 2008.

LIMA, M. G. et al. Fatores de risco para câncer de mama em mulheres indígenas Teréna de área rural, Estado do Mato Grosso do Sul, Brasil. Cadernos de Saúde Pública, Rio de Janeiro, v. 17, n. 5, p. 1537-1544, 2001.

MAJLIS, S. D. Hormonas femeninas y cancer de mama: estado de la polemica y evidencias en 2008. ¿Que responder a las pacientes?. Revista Chilena de Radiologia, Santiago de Chile, v. 14, n. 3, p. 113-121, 2008.

MATOS, J. C. et al. Prevalence of risk factors for breast neoplasm in the city of Maringá, Paraná State, Brazil. Revista Latino-Americana de Enfermagem, Ribeirão Preto, v. 18, n. 3, p. 352-359, 2010.

NATIONAL BREAST AND OVARIAN CANCER CENTRE (NBOCC). Breast cancer risk factors: a review of the evidence. Surry Hills, NSW, Australia: National Breast and Ovarian Cancer Centre, 2009.

NELSON, N. Migrant studies aid the search for factors linked to breast cancer risk. Journal of the National Cancer Institute, Oxford, v. 98, n. 7, p. 436-438, 2006.

PERISSINOTTO, C. et al. Anthropometric measurements in the elderly: age and gender differences. British Journal of Nutrition, Cambridge, v. 87, n. 2, p. 177-186, 2002.

PINHO, V. F. S.; COUTINHO, E. S. F. Variáveis associadas ao câncer de mama em usuárias de unidades básicas de saúde. Cadernos de Saúde Pública, Rio de Janeiro, v. 23, n. 5, p. 1061-1069, 2007.

ROMIEU, I.; LAJOUS, M. The role of obesity, physical activity and dietary factors on the risk for breast cancer: mexican experience. Salud Pública de México, Cuernavaca, v. 51, supl. 2, p. s172-2180, 2009. 
SILVA, E. P. et al. Exploração de fatores de risco para câncer de mama em mulheres de etnia Kaingáng, Terra Indígena Faxinal, Paraná, Brasil, 2008. Cadernos de Saúde Pública, Rio de Janeiro, v. 25, n. 7, p. 1493-1500, 2009.

SILVA, I. S. et al. Lifelong vegetarianism and risk of breast cancer: a population-based case-control study among South Asian migrant women living in England. International Journal of Cancer, Malden, v. 10, n. 99, supl. 2, p. 238-244, 2002.

TESSARO, S. Epidemiologia do câncer de mama. In: BOFF, R. A. (Org.). Mastologia aplicada: abordagem multidisciplinar. Caxias do Sul, RS: Educs, 2001. p. 15-28.

THIRD REPORT OF CHOLESTEROL EDUCATION PROGRAM (NCEP). Expert panel on detection, evaluation, and treatment of high blood cholesterol in adults. NIH Publication, n. 02-5215, 2002. (Adult Treatment Panel III).

SÁNCHEZ, L. et al. Dieta y cáncer de mama en Latinoamérica. Salud Pública de México, Cuernavaca, v. 51, supl. 2, p. 181-190, 2009.

VASCONCELOS, A. B. et al. Height, weight, weight change and risk of breast cancer in Rio de Janeiro, Brazil. São Paulo Medical Journal-Revista Paulista de Medicina, São Paulo, v. 119, n. 2, p. 62-66, 2001.

WEINER, J. G. et al. Analysis of the relationship between diet and exercise beliefs and actual behaviors among breast cancer survivors in Northwest Ohio. Breast Cancer: Basic and Clinical Research, Auckland, v. 4, [s. n.], p. 5-13, 2010.

WORLD CANCER RESEARCH FUND (WCRF). Food, nutrition and prevention of cancer: a global perspective. Washington: American Institute for Cancer Research, 2007.

WORLD HEALTH ORGANIZATION (WHO). Breast cancer: prevention and control. Disponível em: <http://www.who.int/cancer/ detection/breastcancer/en>. Acesso em: 19 jul. 2012. 